\title{
BUILT-IN RESTRICTIONS ON BEST LINEAR UNBIASED PREDICTORS (BLUP) \\ OF RANDOM EFFECTS IN MIXED MODELS 1 /
}

Shayle R. Searle

Biometrics Unit, Cornell University, Ithaca, N. Y.

\begin{abstract}
In the usual mixed model of analysis of variance we show that certain sums of best linear unbiased predictors (BLUP) of random effects are zero. Those sums are similar to, but not exactly the same as, those of the $\Sigma$-restrictions sometimes used for fixed effects.
\end{abstract}

Key words: BLUP, summing to zero, main effects, interactions, fixed effects.

\section{INTRODUCTION}

A frequently-used model equation for a mixed analysis-of-variance model is

$$
\mathbf{y}=\mathbf{X} \boldsymbol{\beta}+\mathbf{Z u}+\mathbf{e}
$$

where $\mathbf{y}$ is a vector of $\mathrm{N}$ observations, $\boldsymbol{\beta}$ and $\mathbf{u}$ are vectors of fixed and random effects, respectively, and e is a vector of residual error terms. $X$ and $Z$ in (1) are known matrices, often incidence matrices (all elements 0 or 1), although they can include columns of observed covariables. The expected value of $y$ is deemed to be $\mathbf{X} \boldsymbol{\beta}$ and that of each of $\mathbf{u}$ and $\mathbf{e}$ is taken to be null. $\mathbf{A}$ broad class of models of this form has two widely-used characteristics: one is that $\beta$ include a general mean $\mu$, and the other is that $\mathrm{Z}$ be an incidence matrix. With these two not-very-restrictive limitations on (1) we establish built-in restrictions on the best linear unbiased predictors (BLUP) of the elements of $u$.

The restrictions are that certain sums of elements of BLUP(u) add to zero. These restrictions are a consequence of the very form of BLUP; they are not a consequence of any definitional restriction such as $\Sigma \alpha_{i}=0$ often seen as part of the model equation $y_{i j}=\mu+\alpha_{i}+e_{i j}$ for the 1-way classification. The restrictions that follow from BLUP are two-fold:

1/ Paper No. BU-734 in the Biometrics Unit. 
(I) For each random effects factor, the sum of the BLUPs of its effects is zero.

(II) For each random effects interaction factor, where the interaction is of a fixed effects factor and a random effects factor, the sum over each level of the fixed effects factor of the BLUPs of the effects of the interaction factor is zero.

An example of (II) is that if $\tilde{\gamma}_{i j}$ is the BLUP of the interaction effect of level $i$ of a fixed effects factor with level $\mathrm{j}$ of a random effects factor then $\Sigma_{j} \tilde{\gamma}_{i j}=0 \forall i$. Note that (II), pertaining as it does to random interaction effects, holds only when those interactions are of fixed effects with random effects, and not of random effects with random effects.

The existence of (I) and (II) has been noted in numerical examples by McLean, Sanders and Stroup (1991, last paragraph, pages 56 and 61). But they give no evidence of the generality of these results, nor do they give any reference thereto. Moreover, a number of personal colleagues to whom these results have been mentioned have commented along the lines of "Oh, that's well known," but no one has been able to provide any references. And, although both (I) and (II) were noted, as above, in McLean et al., neither it nor any other known reference has made the observation contained in the paragraph preceding this one. Derivation of these results therefore follows.

\section{THE USUAL MIXED MODEL and BLUP(u)}

We confine attention to the specific forms of $\beta, \mathbf{X}, \mathbf{u}, \mathbf{Z}, \mathbf{D}$ and $\mathbf{R}$ that occur in the usual mixed model. They are as follows.

(i) $\beta$ has $\mu$, a general mean, as its first element, so that $\beta^{\prime}=\left[\begin{array}{ll}\mu & \beta_{0}^{\prime}\end{array}\right]$ where $\beta_{0}$ is $\beta$ excluding $\mu$.

(ii) Corresponding to (i),

$$
\mathbf{X}=\left[\begin{array}{ll}
\mathbf{1}_{N} & \mathbf{X}_{0}
\end{array}\right]
$$

(iii) $u$ is partitioned as $u^{\prime}=\left[\begin{array}{lllll}u_{1}^{\prime} & u_{2}^{\prime} & \cdots & u_{i}^{\prime} \cdots & u_{r}^{\prime}\end{array}\right]$, where each $u_{i}$ has as its $q_{i}$ elements all the random effects (that occur in the data) corresponding to one random effects factor, be it a main effects factor or an interaction factor.

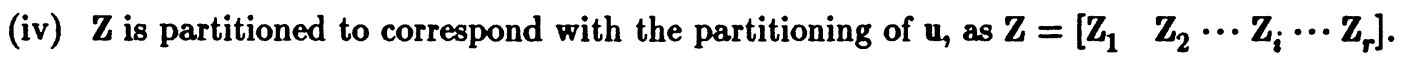

(v) D is a block diagonal matrix of matrices $\operatorname{var}\left(\mathbf{u}_{i}\right)=\sigma_{i}^{2} \mathbf{I}_{i}$ :

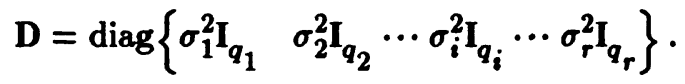

$$
\begin{aligned}
& \mathbf{R}=\sigma_{e}^{2} \mathbf{I}_{N} \text {. }
\end{aligned}
$$


There are many derivations of $\operatorname{BLUP}(\mathbf{u})$ as seen, for example, in Searle et al. (1992, Chapter 7).

The result to be used here is

$$
\operatorname{BLUP}(\mathbf{u})=\tilde{\mathbf{u}}=\mathbf{D Z} \mathbf{P y}
$$

for $D=\operatorname{var}(u)$ of $(4)$, and

$$
\mathbf{P}=\mathbf{V}^{-1}-\mathbf{V}^{-1} \mathbf{X}\left(\mathbf{X}^{\prime} \mathbf{V}^{-1} \mathbf{X}\right)^{-} \mathbf{X}^{\prime} \mathbf{V}^{-1}
$$

with

$$
\mathbf{P X}=\mathbf{0}
$$

and, from (1), (3) and (4)

$$
\mathbf{V}=\operatorname{var}(\mathbf{y})=\mathbf{Z D Z}+\mathbf{R}
$$

\section{A GENERAL RESTRICTION ON BLUP(u)}

A general form of restriction stems from PX $=0$ of (6). For, with that, whenever vectors $\lambda$ and $\tau$ exist such that

$$
\mathbf{Z D} \boldsymbol{\lambda}=\mathbf{X} \boldsymbol{\tau}
$$

then a restriction on $\tilde{\mathbf{u}}$ is

$$
\lambda^{\prime} \tilde{\mathbf{u}}=\lambda^{\prime} \mathbf{D Z} \mathbf{Z}^{\prime} \mathbf{P} \mathbf{y}=(\mathbf{P X} \tilde{\tau})^{\prime} \mathbf{y}=\mathbf{0}
$$

\section{AN EXAMPLE}

Consider the familiar 2-way crossed classification having model equation

$$
\mathrm{y}_{i j k}=\mu+\alpha_{i}+\beta_{j}+\gamma_{i j}+\mathrm{e}_{i j k}
$$

where $\mu$ is a general mean, $\alpha_{i}$ and $\beta_{j}$ are main effects, $\gamma_{i j}$ is an interaction effect and $e_{i j k}$ is a residual

\begin{tabular}{|c|c|c|c|}
\hline & & $\mathbf{n}_{i j}$ & \\
\hline & $\mathrm{j}=1$ & $\mathrm{j}=\mathbf{2}$ & $\mathrm{j}=3$ \\
\hline $\mathbf{i}=$ & 1 & 2 & 4 \\
\hline $\mathrm{i}=\mathbf{2}$ & 3 & - & 2 \\
\hline
\end{tabular}
error. Suppose the $\alpha s$ are fixed effects and the $\beta s$ and $\eta s$ are random with zero means, zero covariances, and variances $\sigma_{\beta}^{2}$ and $\sigma_{\gamma}^{2}$, respectively. In terms of (2) and (3) we write (a) as

$$
\mathbf{y}=1 \mu+\mathbf{X}_{0} \alpha+\mathbf{Z}_{1} \beta+\mathbf{Z}_{2} \gamma+e .
$$

To solidify ideas and illustrate (9) we take as an example the following values of $n_{i j}$, the number of observation in the cell defined by the i'th $\alpha$ and the $j^{\prime}$ th $\beta$, for $i=1,2$ and $j=1,2$, and 3 .

Corresponding to these $n_{i j}$-values the matrices of (11) are 


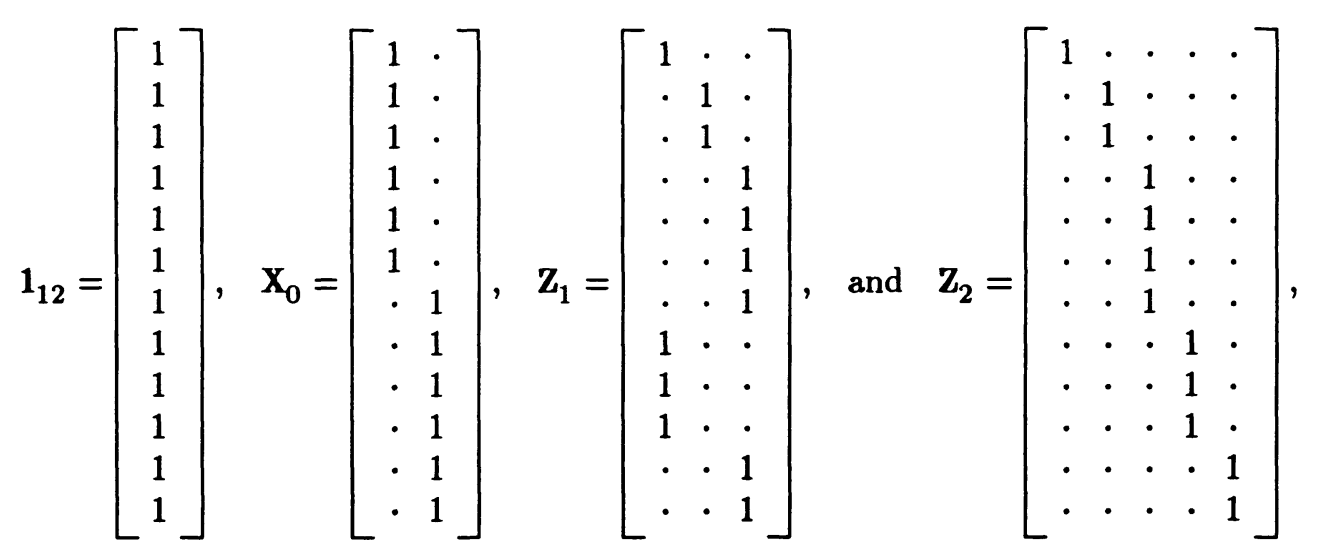

where each dot represents zero. And, with $\mathbf{u}^{\prime}=\left[\begin{array}{ll}\beta^{\prime} & \gamma^{\prime}\end{array}\right]=\left[\begin{array}{llllllll}\beta_{1} & \beta_{2} & \beta_{3} & \gamma_{11} & \gamma_{12} & \gamma_{13} & \gamma_{21} & \gamma_{23}\end{array}\right]$, the matrix $D$ of (4) is

and

$$
\mathbf{D}=\left[\begin{array}{cc}
\sigma_{\beta}^{2} \mathbf{I}_{3} & \cdot \\
\cdot & \sigma_{\gamma}^{2} \mathbf{I}_{5}
\end{array}\right]
$$

$$
\mathbf{Z D}=\left[\begin{array}{lll}
\mathbf{Z}_{1} \sigma_{\beta}^{2} & \mathbf{Z}_{2} \sigma_{\gamma}^{2}
\end{array}\right]
$$

4.1 Random effects factors From (14), (12) and (2), in that order, it is easily seen that

$$
\mathrm{ZD}\left[\begin{array}{c}
1_{3} \\
\mathbf{0}_{5}
\end{array}\right]=\mathrm{Z}_{1} \sigma_{\beta}^{2} 1_{3}=1_{12} \sigma_{\beta}^{2}=\left[\begin{array}{ll}
1_{12} & \mathrm{X}_{0}
\end{array}\right]\left[\begin{array}{l}
1 \\
\mathbf{0}_{2}
\end{array}\right] \sigma_{\beta}^{2}=\mathrm{X}\left[\begin{array}{l}
1 \\
\mathbf{0}_{2}
\end{array}\right] \sigma_{\beta}^{2},
$$

which exemplifies (8) and thus from (9)

$$
\left[\begin{array}{ll}
1_{3}^{\prime} & 0_{5}^{\prime}
\end{array}\right] \tilde{\mathbf{u}}=1_{3}^{\prime} \tilde{\beta}=\sum_{j=1}^{3} \tilde{\beta}_{j}=0 .
$$

Thus is (I) of Section 1 illustrated. And likewise $\Sigma_{j} \Sigma_{j} \tilde{\gamma}_{i j}=0$. This applies for each random effects factor, regardless of whether it is a main effects or an interaction effects factor: the BLUPs of all the effects of each random effects factor sum to zero.

\subsection{Interaction of fixed and random}

Not only do the BLUPs of all the effects of each interaction factor add to zero, as in the preceding sub-section, but they also add to zero in sub-sets, summed over $j$ for each $i$, as in (II). This is illustrated by

$$
\mathrm{ZD}\left[\begin{array}{l}
\mathbf{0}_{3} \\
1_{3} \\
\mathbf{0}_{2}
\end{array}\right]=\mathrm{Z}_{2} \sigma_{\gamma}^{2}\left[\begin{array}{l}
1_{3} \\
\mathbf{0}_{2}
\end{array}\right]=\left[\begin{array}{c}
1_{7} \sigma_{\gamma}^{2} \\
\mathbf{0}_{5}
\end{array}\right]=\left[\begin{array}{ll}
1_{12} & \mathrm{X}_{0}
\end{array}\right]\left[\begin{array}{l}
0 \\
1 \\
0
\end{array}\right] \sigma_{\gamma}^{2}=\mathrm{X}\left[\begin{array}{l}
0 \\
1 \\
0
\end{array}\right] \sigma_{\gamma}^{2},
$$

as an example of (8). Hence from (9)

$$
\left[\begin{array}{lll}
0_{3}^{\prime} & 1_{3}^{\prime} & 0_{2}^{\prime}
\end{array}\right] \tilde{\mathbf{u}}=1_{3}^{\prime}\left[\begin{array}{c}
\tilde{\gamma}_{11} \\
\tilde{\gamma}_{12} \\
\tilde{\gamma}_{13}
\end{array}\right]=\sum_{j=1}^{3} \tilde{\gamma}_{1 j}=0
$$


Thus is (II) illustrated. And $\tilde{\gamma}_{21}+\tilde{\gamma}_{23}=0$ is established similarly, through starting with $\mathrm{ZD}\left[01_{5}^{\prime} \quad \mathbf{1}_{2}^{\prime}\right]^{\prime}$.

\subsection{Generalization}

Although results (I) and (II) have been established only for a simple example, it is clear that they apply quite generally for the usual mixed model described in Section 2. For example, corresponding to $u_{i}$ the equation (9) will hold, in the manner of (16), with the $\lambda^{\prime}=\left[\begin{array}{ll}1_{3}^{\prime} & 0_{5}^{\prime}\end{array}\right]$ vector of (16) being a vector

of order $\sum_{i=1}^{r} q_{i}$ that is null except for $1_{q_{i}}^{\prime}$ in elements $\sum_{t=1}^{i-1} q_{t}+1$ to $\sum_{t=1}^{i} q_{t}$. Likewise for random interaction effects (interactions of fixed with random) the vector post-multiplying ZD in (17) will be null save for an appropriately placed $\mathbf{1}_{m}^{\prime}$, where $\mathrm{m}$ is the number of interaction effects (in the data) whose sum is to be zero; e.g., in (17) $\mathrm{m}=3$, and at the very end of Section $4.2 \mathrm{~m}=2$.

\section{Four important features}

\section{(1) Interactions must be fixed-by-random}

Result (II) occurs only because the $\alpha \mathrm{s}$ in (10) are fixed effects and the interactions denoted by $\gamma$ are interactions of a random factor with that fixed factor. This would not occur if the $\alpha$ s were random, because then the matrix $X_{0}$ would be a $Z$ and the equality in (15) would not exist. Thus (II) applies for random interaction effects only when those interactions are interactions of fixed factors with random factors. It does not apply for interactions that are random-by-random. (I) still applies in that case, but (II) does not.

\section{(2) Empty cells do not affect the result}

Having empty cells means that some $n_{i j}$ are zero, as in the example. This does not affect the argument that leads to (II).

(3) Multi-factor cases

Although (II) has been illustrated in terms of just two factors, its derivation for multi-factor cases would follow the same lines. Thus (II) applies quite generally, to random effect interactions that are interactions of random with fixed factors.

\section{(4) Nested classifications}

Suppose in the example that the $\beta$-factor was not crossed with the $\alpha$-factor but nested within it. Then $Z_{1}$ would not occur and the $\gamma \mathrm{s}$ would be not interaction effects but main effects for the $\beta$-factor 
nested within the $\alpha$-factor levels. All this would mean is that $\tilde{\mathbf{u}}$ of $(9)$ would have no terms in $\beta$, and this affects the arguments leading to (II) not one whit. Thus (II) applies also to random effects nested within fixed effects.

\section{Acknowledgement}

Thanks go to the referee who provided (8) and (9). These lead to the summing-to-zero properties much more easily than does using the mixed model equations [e.g., equations (53) in Chapter 7 of Searle et al. (1992)], as was done in the first draft of this paper.

\section{$\underline{\text { References }}$}

McLean, R. A., Sanders, W. L. and Stroup, W. W. (1991). A unified approach to mixed linear models. The American Statistician 45, 54-64.

Searle, S. R., Casella, G. and McCulloch, C. E. (1992). Variance Components, Wiley, New York. 